\title{
A compact UHV deposition system for in situ study of ultrathin films via hard $x$-ray scattering and spectroscopy
}

\author{
Sebastien Couet, ${ }^{\text {a) }}$ Thomas Diederich, Kai Schlage, and Ralf Röhlsberger \\ Deutsches Elektronen Synchrotron (DESY), Notkestraße 85, 22603 Hamburg, Germany
}

(Received 4 June 2008; accepted 25 August 2008; published online 23 September 2008)

\begin{abstract}
We report on a compact ultrahigh vacuum deposition system developed for in situ experiments using hard $\mathrm{x}$ rays. The chamber can be mounted on various synchrotron beamlines for spectroscopic as well as scattering experiments in grazing incidence geometry. The deposition process is completely remotely controlled and an ellipsometer is available for online monitoring of the layer growth process. The unique sample position in the chamber allows one to perform deposition, grazing incidence x-ray experiments, and ellipsometry measurements at the same time, enabling to correlate the $\mathrm{X}$-ray analysis with parameters of the growth process. Additionally, the setup can be used to study in situ chemical and structural changes in an element specific manner by $\mathrm{x}$-ray absorption spectroscopy. The flexibility and versatility of the system brings new possibilities to study the chemistry and structure of surfaces and interfaces in thin films systems during their formation. (C) 2008 American Institute of Physics. [DOI: 10.1063/1.2982059]
\end{abstract}

\section{INTRODUCTION}

Nowadays, thin films and multilayer structures are used in a large range of applications such as optical coating, microelectronics, ${ }^{1}$ solar cell technology, ${ }^{2}$ or magnetic recording. ${ }^{3}$ Physical vapor deposition techniques such as molecular beam epitaxy or sputter deposition allow for a controlled deposition process and are widely used to grow thin films or multilayer structures. Generally, the properties of the complete system are largely influenced by the quality (structure, chemistry, thickness) of the different interfaces. The study of the growth process during the interface formation is therefore mandatory to tailor the properties of the final device. Usually, an ultrahigh vacuum (UHV) environment is required to study free surfaces, interface formation, or more generally, for any type of in situ study where analysis has to be carried out at selected steps of the preparation procedure. A large variety of analysis technique based on electron probes (Auger spectroscopy, x-ray photoelectron spectroscopy, low energy electron diffraction, etc.) as well as scanning probe microscopy techniques are readily available in common UHV systems. While a combination of these techniques can bring a complete characterization of the chemistry and morphology of thin film surfaces, their probing depth is usually restricted to a few nanometers.

On the other hand, hard $\mathrm{x}$-ray scattering techniques are usually used to probe the structure of bulk material on various length scales in a noninvasive way. The possibility to tune the energy (or wavelength) of the x-ray photons to a particular absorption edge can be used to study chemical and structural parameters of the sample in an element specific manner. ${ }^{4}$ The case of grazing incidence scattering is particularly interesting for the study of thin films and multilayers

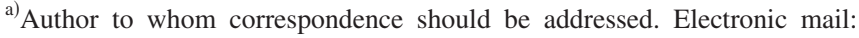
sebastien.couet@desy.de.
}

because this geometry allows to control the penetration depth of the $\mathrm{x}$ rays. Scattering and spectroscopy techniques can therefore be used in a depth resolved manner to study buried layers and interfaces.

Specular x-ray reflectivity can be used to obtain depth resolved density profiles with subnanometer resolution., The analysis of the diffusely scattered radiation at small angles of incidence allows one to obtain information on the lateral ordering of layers or clusters on various length scales. ${ }^{7}$ Resonant techniques such as nuclear forward scattering combined with the use of isotopic probe layers can be used to obtain magnetic depth profiles with sub $\mathrm{nm}$ resolution. ${ }^{8}$

The high intensity provided by modern synchrotron radiation sources enables one to collect data sufficiently fast to perform time resolved studies. In the last decade, the applications of x-ray scattering techniques to follow thin film growth in situ have attracted an increasing interest. In particular, in situ x-ray reflectivity was often used to follow the evolution of the film morphology (roughness, thickness) in real time during growth. ${ }^{9-13}$ In order to perform reflectivity as well as other $\mathrm{x}$-ray scattering experiments, several portable deposition systems have been developed. ${ }^{13-15}$ They usually feature a compact design with large $\mathrm{x}$-ray windows which allows to perform different $\mathrm{x}$-ray scattering experiments such as reflectivity and small angle scattering. They usually accommodate space for Knudsen cells, e-beam evaporators, or sputter guns and have high (sometimes ultrahigh) vacuum capabilities.

In this paper, we describe a compact UHV deposition chamber that was developed to perform grazing incidence scattering and spectroscopy experiments in situ using hard $\mathrm{x}$ rays. The primary goal of this device is to study the growth and the interface formation of transition metal elements $(\mathrm{Fe}$, $\mathrm{Co}$, and $\mathrm{Ni}$ ), as well as oxidation processes on such metallic surfaces. This requires particular care regarding vacuum 

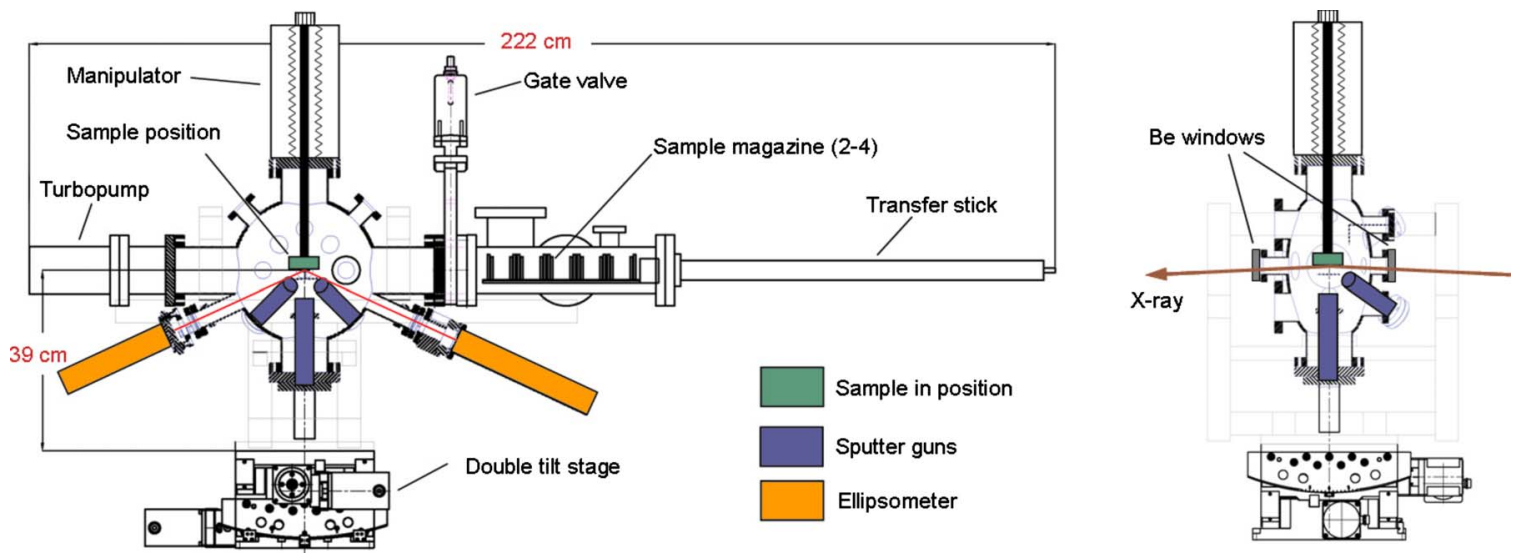

FIG. 1. (Color online) Sketch of the UHV deposition system. The sample is located in the center of the deposition/scattering chamber. Three sputter guns are implemented for deposition of different materials. An ellipsometer attached to the chamber allows one to monitor the growth process during deposition. The sample is in the center of rotation of a two-circle goniometer, allowing for precise angular adjustment with respect to the incoming x-ray beam. A load lock attached to the system is used for fast sample transfer without breaking the main chamber vacuum. The relatively compact dimensions of the system allows one to install it in many experimental stations of present synchrotron radiation sources.

quality and surface modification monitoring. The system has been kept compact to fit in most synchrotron experimental hutches without compromising the experimental flexibility. In particular it is equipped with a load/lock system for fast sample exchange and an ellipsometer for online monitoring of the growth process. The ellipsometry, together with a complete remote control of the deposition process, allows one to precisely follow the evolution of the sample surface during the x-ray scattering experiments. In the first part, the technical design and the general possibilities of the setup are discussed. As an example and a first application of this system, we will present two techniques which were combined to resolve the magnetic and chemical structure of free and buried native iron oxide layers. First, an in situ nuclear forward scattering experiment will demonstrate the possibilities to study magnetic thin film's growth in grazing incidence geometry. The second part is devoted to in situ x-ray absorption spectroscopy (XAS) experiments on ultrathin transition metal layers.

\section{TECHNICAL DESIGN}

One of the main goals was to build a system that is small enough to fit in most of the synchrotron experimental stations yet having enough freedom to keep flexibility in the sample preparation. The main concept relies on the sample position in the center of the chamber which is the same for deposition, grazing incidence x-ray scattering, and ellipsometry measurements, as depicted in Fig. 1. As will be described later, this geometry allows one to perform real-time studies where the deposition takes place at the same time as $\mathrm{x}$-ray scattering data are collected.

The system is composed of a main UHV chamber that is used for the film preparation and the analysis using $\mathrm{x}$ rays, and a load-lock system for fast sample transfer without breaking the main chamber vacuum. As can be seen in Fig. 2 , the system is surrounded by a rigid aluminum cradle (made of aluminum profile Rose-Krieger F50), which can be mounted on a heavy duty two-circle goniometer (maximum load $200 \mathrm{~kg}$, HUBER Diffraktionstechnik GmbH, model
5202.8). This goniometer is equipped with step motors which achieve an angular precision of $10^{-5} \mathrm{deg}$. The center of rotation of this double tilt stage is located at the sample position, $390 \mathrm{~mm}$ above the goniometer plate. As will be described later, precise sample alignment with respect to the x-ray beam is achieved by moving the complete system placed on heavy duty horizontal and vertical $(X-Z)$ translation tables commonly available at synchrotron facilities. This scheme leads to sample positioning precision of few microns with respect to the beam, without affecting the sample position in the chamber. With total dimensions of $222 \times 80 \mathrm{~cm}^{2}$, the system is still compact enough to be mounted on various synchrotron experimental stations.

UHV conditions are reached using a two stage turbomolecular pumping scheme coupled to a membrane pump. This is an oil-free pumping system that can easily reach UHV conditions $\left(<5 \times 10^{-10}\right.$ mbar routinely achieved) and sustain gas injection at moderate pressure $\left(10^{-5}-10^{-2}\right.$ mbar $)$. The pressure in the main chamber is read out by a hot cathode pressure gauge ITR100 from Leybold (pressure range of $\left.1.0 \times 10^{-10}-3 \times 10^{-2} \mathrm{mbar}\right)$. In addition to the flanges used for sputter sources, ellipsometry and gas inlets, 3 CF 40 and 1 CF63 flange can be used to connect other equipments such as additional gas inlet or a mass spectrometer.

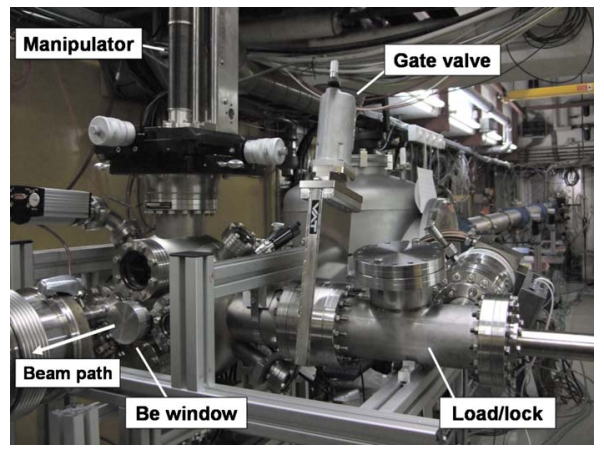

FIG. 2. (Color online) Picture of the deposition system at the BW4 beamline of HASYLAB. The beam enters and exits the chamber in grazing incidence geometry. A protection cap is placed on the Be exit window. 
The load lock is equipped with a turbopump connected to another membrane pump. When high vacuum is reached in the load-lock chamber $\left(p<1 \times 10^{-5} \mathrm{mbar}\right)$, the membrane pump is disconnected to allow the load/lock turbopump to be connected to the second turbomolecular pumping stage. This way, pressures below $1 \times 10^{-8}$ mbar can be reached in the load lock without the need for a baking out procedure. A sample magazine containing a maximum of four samples is available and transfer to the main chamber is done through a large CF100 gate valve. The details of the sample transfer system are given in Sec. II A. Two entrance and exit beryllium (PF-1 grade, Brush Wellman Inc.) windows for hard $\mathrm{x}$ rays can be used. They are $3.5 \mathrm{~cm}$ in diameter (placed on a CF40 flange) and have a thickness of $120 \mu \mathrm{m}$. This small thickness, together with the high purity grade, is chosen to avoid parasitic signal from eventual traces of heavy elements. In particular, the low concentration in Fe is particularly suited for nuclear forward scattering experiments on ${ }^{57} \mathrm{Fe}$ nuclei where any traces of Fe along the path of the $\mathrm{x}$-ray beam lead to background signal. The transmission through this type of window is as high as $70 \%$ for energies above 3.5 $\mathrm{keV}$. Moreover, the windows do not modify the beam profile significantly, as evidenced in a recent grazing incidence small angle x-ray spectroscopy (GISAXS) experiment. ${ }^{16}$

The system can be mounted on the beamline in 1 day while proper bakeout and cool down procedure requires another 3 days. For that reason, the setup is usually mounted in an unused experimental hutch while other experiments can be carried out in another experimental hutch of the beamline. If this scheme is not applicable, mounting the system during a longer shutdown period of the synchrotron (such as maintenance week) is usually preferred to maximize the use of the photon beam.

\section{A. Sample environment}

The main specifications for the sample holder system are imposed by the grazing incidence geometry used. The $\mathrm{x}$-ray beam usually exhibits a vertical beam size in the range of 30-60 $\mu \mathrm{m}$. Because the beam impinges on the surface at grazing angles (usually below $1^{\circ}$ ), this leads to a beam footprint size on the sample of 10-20 mm. In order to collect the full signal and avoid parasitic scattering from the sample edges, this requires samples of similar dimensions. The sample holder was therefore designed to accommodate relatively large samples and provide a completely free pathway for the $\mathrm{x}$-ray beam in grazing incidence.

Three different types of sample holders are available. The first one is designed to fit samples as large as 25 $\times 40 \mathrm{~mm}^{2}$. It can be used in any case where parasitic scattering from the sample holder edges has to be avoided, like in GISAXS. The second type of sample holder was developed to perform magnetic studies. It provides a permanent magnetic field of $70 \mathrm{mT}$ in the plane of the sample. As shown on Fig. 3(a), it consists of hard $(\mathrm{NdFeB})$ and soft $(\mathrm{Fe})$ magnetic material and the design was optimized to provide better than $3 \%$ field homogeneity over the sample surface $\left(20 \times 15 \mathrm{~mm}^{2}\right)$. Finally, a sample holder equipped with a specially designed heater stage was developed to perform temperature dependent studies. As depicted in Fig. 3(b), the
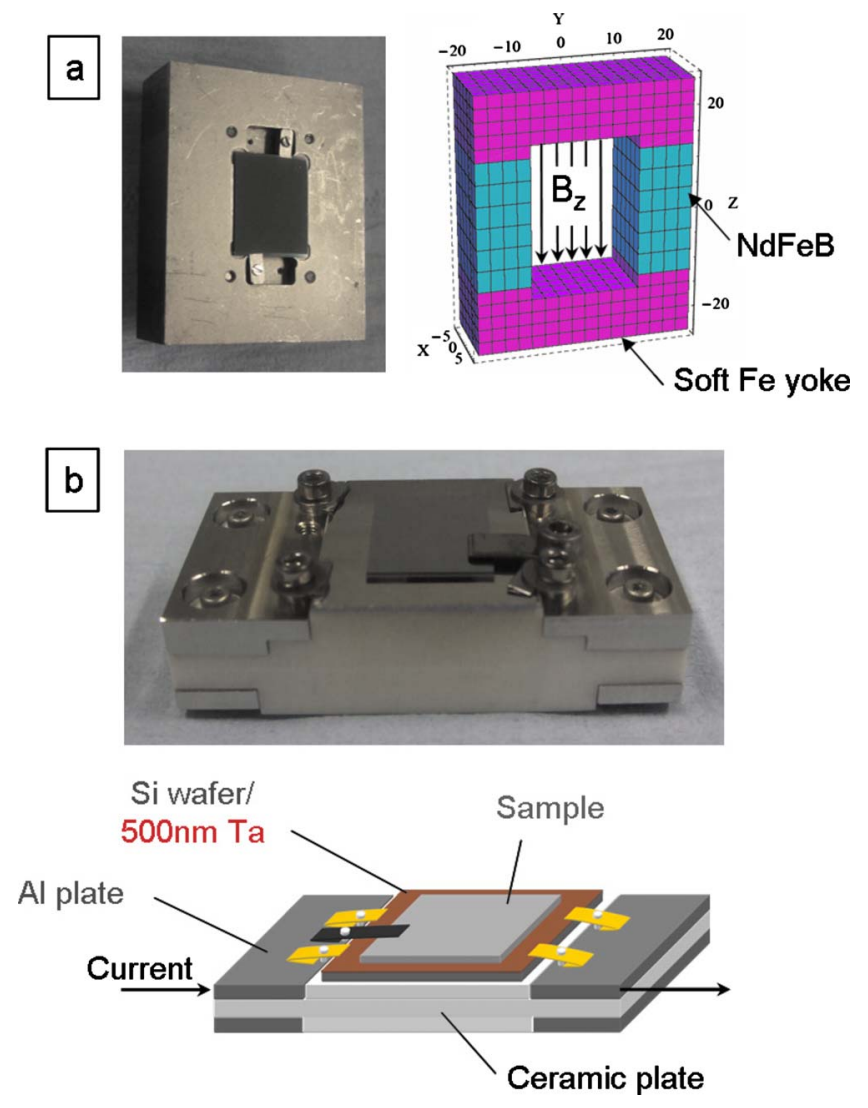

FIG. 3. (Color online) (a) Picture of the magnetic sample holder. The right part displays a schematic of the magnet configuration leading to an in-plane permanent magnetic field of $70 \mathrm{mT}$. (b) Picture and principle diagram of the heater. The sample is resistively heated via current flow through a $500 \mathrm{~nm}$ thick Ta layer on a Si wafer. The temperature is measured by a PT100 temperature sensor placed on the backside of the Si wafer.

sample is placed on a Si wafer coated with a $500 \mathrm{~nm}$ thick Ta layer. Heating is achieved by feeding a current of a few amperes through the Ta layer. This particular design allows for fast heating (up to $600 \mathrm{~K} / \mathrm{min}$ ) and cooling of the sample. The temperature is measured by a UHV compatible PT100 temperature sensor placed on the backside of the Si wafer. Temperature stability better than $0.1 \mathrm{~K}$ is achieved by proportional integral derivative regulation of the heating current. In situ temperature dependent $\mathrm{x}$-ray experiments have not yet been carried out, but it is expected that the temperature stability achieved here should ensure stable experimental conditions once thermal equilibrium is reached.

In all cases, the holder is placed on a three axis rotary manipulator that allows a positioning of the sample in the center of the chamber with $0.1 \mathrm{~mm}$ accuracy. The position of the sample surface with respect to the center of rotation was determined by placing the sample in the center of the entrance window. This position can be easily reproduced upon sample change by controlling the position of the reflected laser beam of the ellipsometer from the sample surface. The position of the center of the window above the goniometer plate is then calculated to be in the center of rotation of the goniometer. The rotation of the sample enables one to perform scattering experiments along different in-plane orientations of the sample.

Two different sample transfers are available. In both 
a)

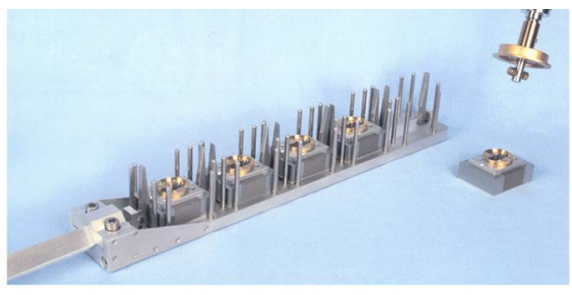

b)

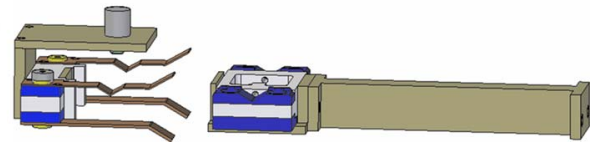

FIG. 4. (Color online) (a) Picture of the sample transfer system for room temperature sample holder. The sample is attached to the manipulator from the backside using a bayonet locking system. (b) Schematic drawing of the transfer system for the heater stage. The two clamps provide electrical contact for the heating plate and the PT100.

cases, the sample is slid horizontally from the load lock using a transfer stick. For the room temperature holders, the manipulator attaches to the backside of the holder using a bayonet system, as shown on Fig. 4(a). For the heater stage, a two fork clamping system is used on the manipulator. The two forks are used to provide electric contact to the heating plate as well as for the PT100, as shown on Fig. 4(b). Typical time needed to transfer a sample from the load lock to the main chamber is 1 min while the load lock can be pumped to sufficiently low pressure to allow sample transfer in less than an hour.

\section{B. Deposition system}

The basic setup of the chamber provides three conflat flanges (one CF100 at the bottom and two CF40 on the sides) centered on the sample position. While several different deposition systems can be implemented (such as Knudsen cells or electron beam evaporators), dc magnetron sputter deposition is usually preferred here for its pronounced advantages. For a given material, the deposition rate is a function of the argon gas pressure and of the power set point of the source only. The growth rate and the energy distribution of the sputtered atoms can be adjusted by controlling those parameters. ${ }^{17}$ This gives the opportunity to adjust the morphology of the deposited films. For example, at high Ar pressure the sputtered atoms are thermalized and cluster growth is promoted. Second, the layers produced are homogeneous over areas of several square centimeters (10\% thickness variation on a $50 \mathrm{~mm}$ diameter film). These are necessary conditions in view of the x-ray grazing incidence experiments. The deposition process is fast: a complete deposition cycle can be achieved in less than $1 \mathrm{~min}$, and allows maximizing the measuring time during the experiment. This type of system can be easily computer controlled, as will be shown in Sec. IID. Finally, dc magnetron sputter deposition is a widely used deposition process in the industry. This setup can therefore be used to mimic industrial processes. The chamber is usually equipped with three dc magnetron sputter sources. The main deposition source is placed below the sample (normal incidence) at a distance of $63 \mathrm{~mm}$ from the sample and fits a $33 \mathrm{~mm}$ diameter target. At this targetsample distance, the homogeneity of the film is better than $10 \%$ on a $50 \mathrm{~mm}$ diameter sample. Two other sources $(25.4$ $\mathrm{mm}$ target) are placed on both sides of the sample with an incidence angle of $35^{\circ}$ relative to the sample plane. While the oblique incidence prevents the formation of homogeneous layer, it can be used, for example, to imprint magnetic anisotropies in transition metal layers ${ }^{18}$ or to deposit a protection layer before bringing the sample back to atmosphere. The deposition of a wedge layer can also provide a means to produce a thickness gradient in the sample. The three sputter guns are equipped with motorized shutters to precisely control the deposition time (opening time $<0.5 \mathrm{~s}$ ). Argon gas ( $99.9999 \%$ purity) is admitted using a mass flow controller which ensures stability and reproducibility of the Ar pressure better than $1 \%$ over a pressure range from $3 \times 10^{-3}$ to $3 \times 10^{-2}$ mbar.

Additionally, a step motor driven leak valve is mounted on the chamber to admit various gases in the pressure range from $5 \times 10^{-8}$ to $5 \times 10^{-3}$ mbar. As will be shown later, it can be used to perform defined oxidation processes by precisely controlling the total oxygen exposure.

\section{Ellipsometry}

As described above, the chamber is equipped with an ellipsometer used here to monitor the layer growth online. Ellipsometry is based on the measurement of the change in polarization state of visible light upon reflection from a medium. The fundamental ellipsometry parameters (that are measured) are $\Delta$ (intensity ratio) and $\Psi$ (phase shift) and can be directly related to the ratio of $R^{p}$ and $R^{s}$, the reflection coefficients for $s$ and $p$ polarized light, respectively, ${ }^{19}$

$$
\frac{R^{p}}{R^{s}}=\tan \Psi e^{i \Delta} .
$$

Since the reflection coefficients depend on the thickness and refractive index of the layer, measuring the change in $\Delta$ and $\Psi$ during the deposition allows one to evaluate the thickness and composition of the layer being grown. We use an ELX-1 ellipsometer (Dr. Riss Ellipsometer Bau GmbH, Germany) equipped with a He-Ne laser emitting at $632.8 \mathrm{~nm}$. The laser and detector are attached to the chamber symmetric to the sample position and perpendicular to the x-ray scattering plane, as shown on Fig. 1. The incidence and exit angle is fixed to $65^{\circ}$ with respect to the sample surface. The determination of $\Delta$ and $\Psi$ is performed by rotation of a quarter wave plate and an analyzer (placed in front of the detection diode) to minimize the transmitted intensity. A measurement cycle can be performed at $1 \mathrm{~Hz}$ repetition rate.

Figure 5 shows the ellipsometry curve for a typical multilayer structure grown during our experiments. In this case, a $2.5 \mathrm{~nm}$ thick Fe layer is sputter deposited at a growth rate of $0.1 \mathrm{~nm} / \mathrm{s}$. The layer is subsequently oxidized by controlled exposure to oxygen. A second $\mathrm{Fe}$ layer and an $\mathrm{Al}$ capping layer are subsequently deposited. Ellipsometry data are acquired continuously during the deposition. The growth of each layer corresponds to characteristic variation of $\Delta$ and $\Psi$ in the ellipsometry curve. Modeling of the multilayer a posteriori allows one to relate the change in $\Delta$ and $\Psi$ to the 


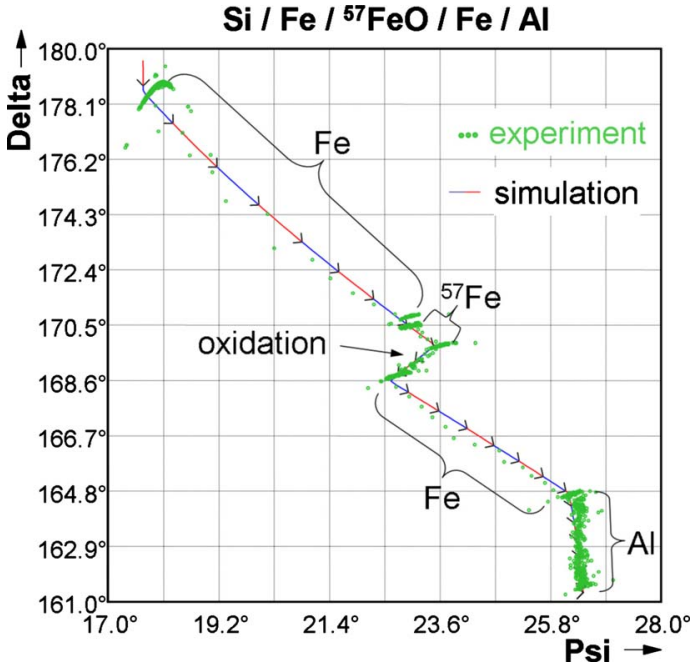

FIG. 5. (Color online) Ellipsometry curve of the growth of a $\mathrm{Si} / \mathrm{Fe}(2.2 \mathrm{~nm}) /{ }^{57} \mathrm{Fe}-\mathrm{O}(1.5 \mathrm{~nm}) / \mathrm{Fe}(2.2 \mathrm{~nm}) / \mathrm{Al}(3 \mathrm{~nm})$ multilayer. The growth of each different layer shows a distinct evolution of the ellipsometry parameters $\Delta$ and $\Psi$. The simulation allows one to estimate the refractive index and the thickness of each layer

refractive index and the thickness of each layer. This model can subsequently be used to monitor the growth process and determine when a certain layer thickness has been deposited. In the case of films below $5 \mathrm{~nm}$, the precision is sufficient to measure monolayer coverage. Above that thickness the sensitivity progressively decreases due to the limited probing depth of light in metals. Furthermore, the technique is highly sensitive to oxidation processes on the surface and can be used to monitor eventual surface chemistry changes during an ongoing x-ray analysis. This is an important aspect either to monitor beam damage in the case of soft substrate (polymer), or to monitor adsorption of molecules due to the rest gas pressure on reactive surfaces.

As mentioned earlier, the ellipsometer is also used to check the position of the sample in the center of the chamber. Because the photodiode as a small entrance window of $2 \mathrm{~mm}$ diameter only, the position of the reflected beam is highly sensitive to the height of the sample in the chamber. Checking the reflection, one can easily reproduce the same sample position upon sample change.

\section{Remote control system}

For an x-ray experiment, the UHV system will be mounted inside the experimental hutch which will not be accessible during the measurement. To provide maximum flexibility and allow for real-time (deposition plus analysis) experiments to be performed, the deposition is completely remote controlled from outside the experimental hutch.

The different equipments operate using different communication protocols (analog signals for the sputter sources, RS232 for mass flow controller and pressure gauge, etc.). An interface box containing all the necessary converters (analog to digital and digital to analog converters, digital input output, relays, etc.) has therefore been designed to merge all the required connections to one single input/output USB port. The complete setup is then controlled using a LABVIEW program running on a remote laptop computer. This flexible solution is easily transportable and completely independent of the different computer systems used at the various synchrotron facilities. The control program presents itself as a single window graphical user interface (GUI), as shown in Fig. 6. Each instrument can be operated independently or at the same time with a continuous monitoring of all the relevant parameters such as pressure, mass flow controller set point, current, voltage, and power of the sputter sources. This gives full flexibility and security over the preparation procedure. Additionally, special procedures have been implemented to perform automatized tasks. The "oxidation cycle" routine can, for example, be used to perform a controlled oxidation of the deposited layer. In that case, the gas pressure is recorded as a function of time ( $10 \mathrm{~Hz}$ sampling rate). The pressure is then integrated over time, which allows one to calculate and precisely control the total exposure (pressure $\times$ time) of the sample to the gas. This can be used to perform oxidation cycles with exposure reproducibility better than $1 \%$. The two parameters that have to be provided are therefore the gas pressure and total exposure desired. Additionally, a deposition sequence can be programed to grow complex multilayer or superlattice structures.

\section{X-RAY SCATTERING TECHNIQUES TO STUDY THE GROWTH OF THIN METAL AND OXIDE FILMS}

The general geometry for grazing incidence experiments is depicted on the right side of Fig. 1. This is a vertical scattering geometry where the $\mathrm{x}$-ray beam is reflected downward. The two Be windows are placed on each side of the chamber to let the x-ray beam in and out in grazing incidence geometry. The distance from the center of the sample to the entrance or exit window is fixed to $17.5 \mathrm{~cm}$ which therefore allows measurements up to incident angles of $\theta=4^{\circ}\left(2 \theta=8^{\circ}\right)$.

In the following, we describe an experiment that aimed at the determination of the evolution of the magnetic structure of buried native iron oxide layers during their formation. This type of ultrathin oxide layers (produced by exposure of a metal substrate to molecular oxygen) embedded in its metal counterpart are expected to exhibit structural and magnetic properties that deviate strongly from any bulk iron oxide phases. In particular, an anomalously high magnetic moment per atom ${ }^{20}$ as well as an antiferromagnetic ordering at room temperature ${ }^{21}$ were reported recently. In order to unravel how such magnetic properties evolve in buried native oxide layers, we studied the formation of these layers in situ by nuclear forward scattering. For an overview of the experimental technique, see Ref. 22 and references therein. The sensitivity of the method to the magnetic structure of buried layers combined with the possibility to completely isolate the oxide signal from the surrounding metallic $\mathrm{Fe}$ by using isotopic probe layers render this technique an ideal candidate for this type of investigations. This experiment is a stepwise experiment where $\mathrm{x}$-ray scattering is performed after each deposition/oxidation and was for the first time made possible thanks to this setup.

The experiment was performed at the nuclear resonance beamline ID18 of the European Synchrotron Radiation 


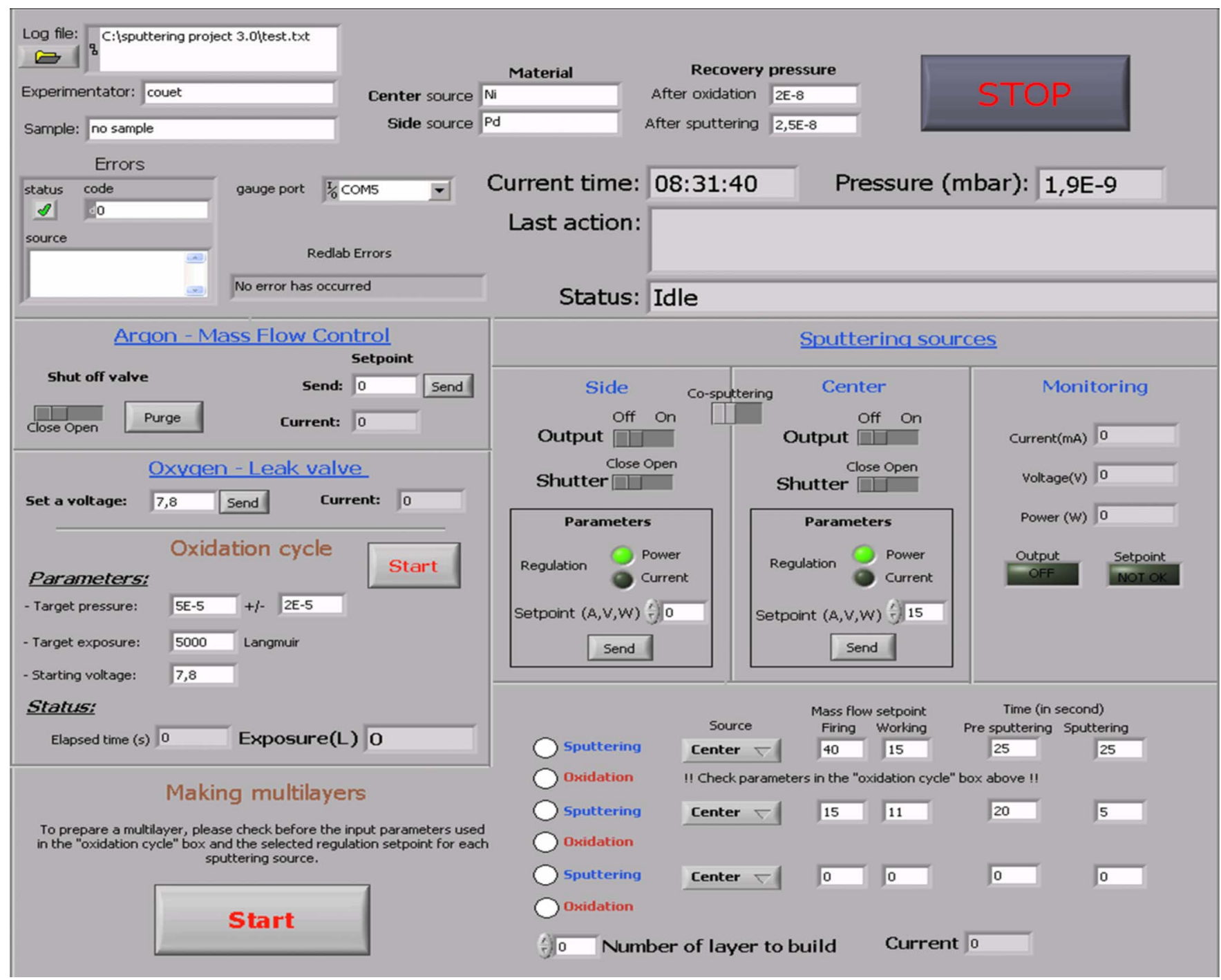

FIG. 6. (Color online) GUI of the LABVIEW based control program for the deposition process. Separate control and monitoring is available for each sputter sources and gas inlets. A sequence of deposition and gas exposure step can be programed to grow multilayer structures consisting of metal and oxide films.

Facility (ESRF). ${ }^{23}$ The UHV system was placed on the double tilt stage referenced in Fig. 1. To precisely position the sample relative to the incoming $x$-ray beam, the whole system was placed on an $X-Z$ table (lateral + vertical movement which allowed alignment of the sample with respect to the $\mathrm{X}$-ray beam with a few micron accuracy). The beam was focused to $30 \mu \mathrm{m}$ in the vertical direction using a Kirkpatrick-Baez mirror to match the sample cross section in grazing incidence geometry. The substrate [superpolished $\mathrm{Si}(100)$ wafer] was placed on a magnetic sample holder to fix the magnetization direction. In order to study exclusively the magnetic properties of the native oxide, we deposited an ultrathin $(0.6 \mathrm{~nm}){ }^{57} \mathrm{Fe}$ probe layer on top of a $2 \mathrm{~nm}$ thick Fe layer. The layer was then oxidized to saturation and stepwise covered with $\mathrm{Fe}$. The experiment was performed at a fixed incidence angle $\theta$ of $0.24^{\circ}$, which is well above the critical angle of $\mathrm{Fe}\left(0.21^{\circ}\right)$ at $14.4 \mathrm{keV}$. At each preparation step, nuclear time spectra (the temporal evolution of the nuclear decay after pulsed excitation) were recorded to follow the evolution of the magnetic structure.

Figure 7(a) shows the time spectra corresponding to the pure $0.6 \mathrm{~nm}$ thick ${ }^{57} \mathrm{Fe}$ layer deposited on top of the $2 \mathrm{~nm}$ thick ${ }^{\text {nat }} \mathrm{Fe}$ layer. The fast beating pattern corresponds to a hyperfine field $B_{\mathrm{HF}}$ of $32 \mathrm{~T}$, a typical value for ferromagnetic $\alpha$-Fe at room temperature. The shape of the spectra indicates that the magnetization is oriented in the plane of the sample parallel to the incoming photon wavevector and the external field. After exposure to $6500 \mathrm{~L}\left(1 \mathrm{~L}=10^{-6}\right.$ Torr s) of oxygen, the ${ }^{57} \mathrm{Fe}$ probe layer is completely oxidized and the fast beating is replaced by a long period oscillation [Fig. 7(b)] due to residual quadrupole interactions in the oxide. The small hyperfine field associated with this beating indicates that the free native oxide is in a superparamagnetic or nonmagnetic state. Subsequent deposition of Fe [Figs. 7(c)-7(e)] leads to a reappearance of a fast beating, indicating a progressive magnetic ordering of the oxide layer. While chemical changes in the oxide are expected upon deposition of $\mathrm{Fe}$, the progressive magnetic transition suggests that the magnetism in the buried native oxide is supported and stabilized by the surrounding metallic Fe. Complementary results can be found in Ref. 21.

The experiment shows the potential to perform in situ 


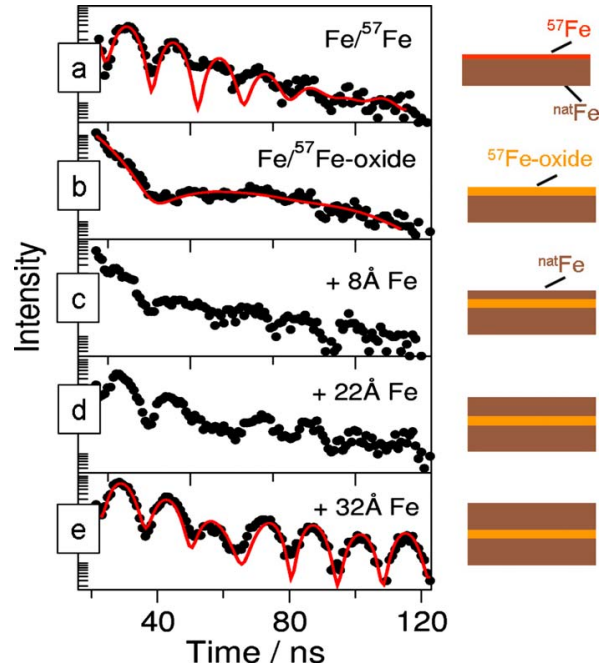

FIG. 7. (Color online) Results of a nuclear forward scattering experiment where an ultrathin ${ }^{57} \mathrm{Fe}$ probe layer is used to resolve the magnetic structure of a buried native oxide layer at different steps of the growth. (a) Time spectra of the pure $0.6 \mathrm{~nm}$ thick ${ }^{57} \mathrm{Fe}$ probe layer placed on top of a $2 \mathrm{~nm}$ ${ }^{\mathrm{Nat}} \mathrm{Fe}$ layer. The fast beating is related to the ferromagnetic ordering of $\alpha$-Fe. (b) Oxidized ${ }^{57} \mathrm{Fe}$ oxide. The large period beating indicates a nonmagnetic state. [(c)-(e)] Progressive reappearance of the beating pattern with increasing $\mathrm{Fe}$ coverage, sign of a recovery of the magnetic order.

$\mathrm{x}$-ray scattering experiments, as it is the only way to observe changes in buried layers during the deposition. In this case, the use of isotopic probe layers allows one to access the magnetic properties of the sample in a layer specific manner. Because of its relation with oxidation process on Fe surfaces, it requires a sufficiently clean environment to avoid contamination during the $\mathrm{x}$-ray analysis (typically $30 \mathrm{~min}$ acquisition time). The continuous ellipsometer analysis confirms that the surfaces do not suffer from the long x-ray exposure or from the residual gas pressure. This experiment was made possible for the first time with this setup.

The ability to measure GISAXS was also demonstrated recently. The experiment, performed at the beamline BW4 of the DORIS III storage ring (Hamburg, Germany), showed the possibility to use in situ GISAXS to study metal growth on polymer surfaces. ${ }^{16}$ In that case, gold was stepwise deposited on a mixed diblock-triblock copolymer and GISAXS patterns were taken at selected gold coverages. This allowed evidencing a progressive diffusion of the gold particles into the polymer matrix and a preferred agglomeration of gold clusters around one of the two polymer chains. This type of experiments can also be coupled with the ellipsometry measurement, providing a unique picture of the optical properties as well as the microscopic structure of the film in real time.

Another possible approach is to record GISAXS patterns during the growth. Preliminary experiments carried out at the BW4 beamline ${ }^{24}$ have shown that GISAXS patterns with sufficiently high statistics can be acquired within $1 \mathrm{~s}$ with this setup. The development of fast read-out large area single photon counting detectors based on hybrid pixel technology ${ }^{25}$ opens the possibility to record GISAXS pattern in only a few ten ms.

\section{X-RAY SPECTROSCOPY OF ULTRATHIN FILMS}

Soft XAS is already a widely used technique to probe chemistry and magnetism of thin films in situ. In this energy

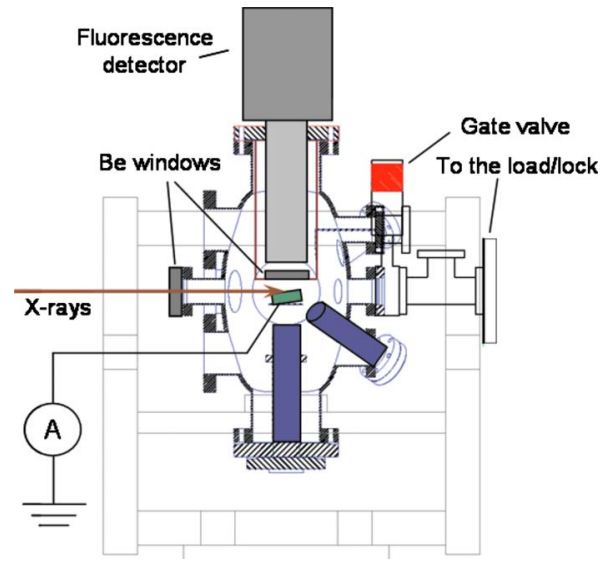

FIG. 8. (Color online) Sketch of the setup for x-ray absorption experiment. Compared to the setup presented in Fig. 1, the manipulator is placed in horizontal position. The incidence angle is therefore adjusted manually. The load lock is connected to the main chamber through a smaller CF40 gate valve. This allows placing the fluorescence detector in vertical geometry $4 \mathrm{~cm}$ above the sample. The TEY is recorded by measuring the drain current on the sample using a low current electrometer (Keithley 4517A).

range however, the resonant absorption usually leads to a strong reduction in the probing depth. This results in complicated data analysis in the case of depth sensitive experiments. In addition, when the fluorescence signal is measured, it is often integrated over the energy. This prevents, for example, to isolate the extended fine structure part of the signal (which usually extents up to $800 \mathrm{eV}$ above the edge). These problems are overcome in the case of hard XAS where energy dispersive fluorescence detectors are generally available.

A classical hard x-ray absorption experiment takes place in transmission geometry where the intensity of the incident and transmitted $\mathrm{x}$-ray beam is measured as a function of the photon energy. However, thin films are usually grown on thick, absorbing substrates (such as silicon or glass) and the transmission geometry is not applicable.

Two other detection schemes can be used for thin film studies: the fluorescence yield (FY) and the total electron yield (TEY). The FY measures the fluorescent photons reemitted by the absorbing atoms. This is an energy dispersive single photon counting measurement which allows one to isolate the contributions from different elements. The technique has a large probing depth (limited only by the attenuation of $\mathrm{x}$ ray in matter) but suffers from self-absorption effects $^{26}$ in concentrated samples. On the other hand, the measure of the TEY provides an energy integrated signal that is intrinsically surface sensitive. It is based on the measure of the drain current on the sample which is induced by the photoabsorption. The usual probing depth of the method is determined by the average mean free path of the electrons in the sample and is estimated to be around $50-100 \mathrm{~nm}$ for excitation with hard $\mathrm{x}$ rays. ${ }^{27}$

These two systems are therefore complementary: FY can be element selective while TEY provides the desired surface sensitivity. Both detection schemes were implemented on the chamber. As the fluorescent photons are emitted isotropically, the detector has to be placed as close as possible to the sample to cover a sufficiently large solid angle. As shown in 

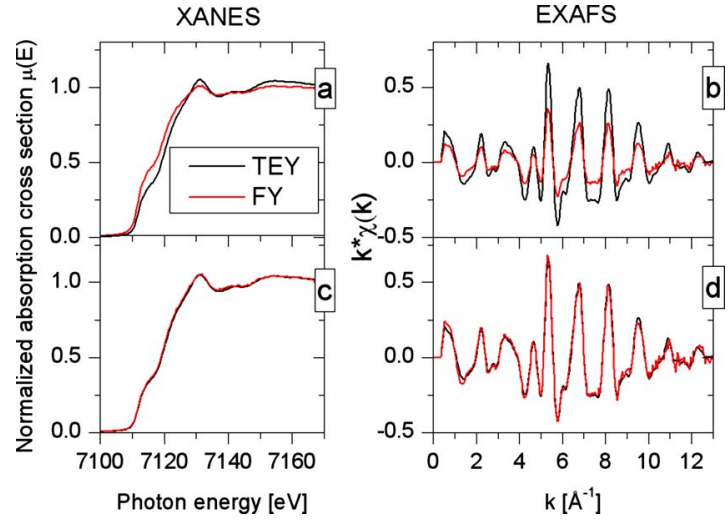

FIG. 9. (Color online) Comparison of the TEY and FY signals of a $30 \mathrm{~nm}$ thick Fe layer. The incidence angle was $20^{\circ}$. (a) Normalized XANES spectra. (b) Weighted EXAFS spectra. [(c) and (d)] Results after correction of the FY for self absorption: the two signals match almost perfectly.

Fig. 8, the geometry of the experiment has to be changed compared to the grazing incidence case. The sample manipulator is now placed horizontally, the incidence angle of the $\mathrm{x}$ rays with respect to the sample will therefore be adjusted manually. The load lock is now connected to the chamber through a CF40 gate valve. The sample holding system has been modified to accommodate for the new geometry. In particular only one sample can be placed in the sample magazine. The free CF100 flanges on the top of the chamber enables the use of a special tube equipped with a $\mathrm{Be}$ window which allows to bring the external Ge detector (Canberra) as close as $40 \mathrm{~mm}$ to the sample surface. The TEY is measured by isolating the sample from the ground and connecting it to a Keithley low current electrometer (Keithley 4517A) using shielded cables and standard safe high voltage feedthrough.

We tested the performance of the two detection systems at the A1 beamline of the DORIS III storage ring (Hamburg, Germany). As a reference layer, we deposited a $30 \mathrm{~nm} \mathrm{Fe}$ layer onto a Pd coated Si wafer. Both the x-ray appearance near edge structure (XANES) and extended x-ray absorption fine structure (EXAFS) of the $\mathrm{Fe} K$-edge $(7112 \mathrm{eV})$ were recorded up to $700 \mathrm{eV}$ above the edge. The normalized spectra are shown in Figs. 9(a) and 9(b). It is directly seen that the TEY provides a much better signal to noise ratio. The result for TEY is remarkable with respect to the small current measured: the background current is around $30 \mathrm{pA}$ and the edge jump is around $300 \%$ of this value $(5 \%$ for a $0.4 \mathrm{~nm}$ thick layer). The observed noise in the EXAFS oscillation accounts for a signal stability of few tens of femtoampere. It is a remarkable result in regards of the relatively simple detection scheme used. However, the TEY and FY spectra do not exhibit the same shape. This is especially seen in the EXAFS signal where the oscillation amplitude of the FY is reduced. This behavior is typical for self-absorption effects which seem to be significant even in the case of thin (but dense) films. Proper corrections for self-absorption were done using the algorithm developed by Booth and Bridges ${ }^{28}$ which was implemented in the ATHENA analysis software. ${ }^{29}$ As can be seen in Figs. 9(c) and 9(d), correction for selfabsorption leads to an almost perfect matching of the two signals. This means that both the energy resolved (FY) and
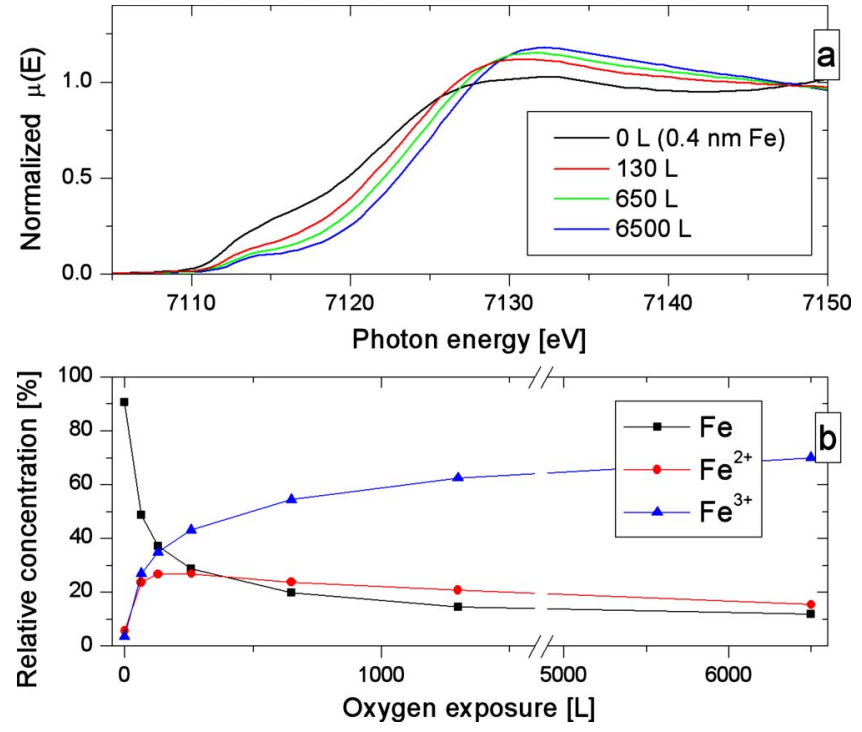

FIG. 10. (Color online) (a) XANES spectra at selected step of the oxidation of a $0.4 \mathrm{~nm}$ thick Fe layer. The shift of the edge toward higher energy indicates an increase in the oxidation stage. (b) Relative concentration of the different oxide species as a function of the oxygen exposure. The growth law for $\mathrm{Fe}^{2+}$ differs from the general growth law for oxide.

energy integrated (TEY) signals provide the same information in the case of thin films. A comparison of both signals should lead to proper correction of self-absorption effect in FY. Due to better statistical quality, the TEY signal should be used whenever energy discrimination is not required.

As a first experiment with this setup, we followed the initial stages of the oxidation process of an ultrathin $\mathrm{Fe}$ layer. We deposited a $0.4 \mathrm{~nm}$ thick Fe layer onto a Pd coated $\mathrm{Si}$ substrate and performed a stepwise oxidation at an oxygen pressure of $1 \times 10^{-5} \mathrm{mbar}$. The corresponding XANES spectra are shown in Fig. 10(a). Starting with the pure Fe layer, we observe a gradual shift of the edge toward higher energies with increasing oxygen exposure, indicating an increase in the overall oxidation state of the $\mathrm{Fe}$ atoms up to saturation at $6500 \mathrm{~L}$. Using a linear combination of reference spectra of the pure oxide compounds, it is possible to extract the relative concentration of each chemical sites. The results are shown in Fig. 10(b). One observes that the growth differs for $\mathrm{Fe}^{2+}$ and $\mathrm{Fe}^{3+}$ type of sites, especially that $\mathrm{Fe}^{2+}$ is created only at the early stage of the oxidation, when the Fe supply is sufficient. A more detailed study of these results will be published elsewhere. ${ }^{30}$

We see that the extreme stability of the TEY signal allows one to record hard x-ray XAS spectra in the monolayer regime, which would become even more advantageous at third generation synchrotron sources. In particular, this opens interesting perspectives for time resolved $\mathrm{x}$-ray absorption studies on thin films growth or interface formation in heterostructures and multilayers.

\section{CONCLUSIONS AND PERSPECTIVES}

We have described a compact UHV setup which can be used for in situ studies via x-ray scattering and spectroscopy. The sample position in the main chamber allows simultaneously performing deposition, ellipsometry measurement, 
and grazing incidence x-ray scattering. This offers unique possibilities to study in situ thin film growth, magnetic transitions in thin films, cluster growth on surfaces, and interface formation. The instrument described here has enabled us to gain insight into thin film growth processes that would not have been possible otherwise. The possibility to perform XAS with the same deposition setup allows one to look at different aspects of the same system. In the case of the XAS experiment, the development of an efficient TEY detection scheme capable of measuring ultrathin films with good signal stability opens interesting possibilities to perform time resolved XAS studies. The examples presented here demonstrate that both grazing incidence scattering and absorption spectroscopy can be performed in separate experiments. A unique application of this setup would be to combine these techniques in a single experiment on a beamline that combines both techniques. This scheme would, for example, allow direct correlation between density depth profile and chemistry at selected steps of the growth of a multilayer structure. Together with ellipsometry, this scheme can provide a link between optical properties and microscopic properties of surfaces and buried layers, a key role in the development of polymer/metal systems. A future development of the experimental setup will include a complete temperature control of the sample from the frontend GUI, allowing controlled deposition process at elevated temperature.

\section{ACKNOWLEDGMENTS}

The authors gratefully acknowledge R. Rüffer and S. Stankov from the ID18 beamline for there help during the NFS experiments as well as E. Welter and D. Zajac from HASYLAB for assistance during the x-ray absorption experiment. This work was supported by the European Commission under FP6 Contract No. NMP4-CT-2003-001516 (DYNASYNC).

\footnotetext{
${ }^{1}$ S. J. Pearton, F. Ren, A. P. Zhang, and K. P. Lee, Mater. Sci. Eng., R. 30, 55 (2000).

${ }^{2}$ K. L. Chopra, P. D. Paulson, and V. Dutta, Prog. Photovoltaics 12, 69 (2004).

${ }^{3}$ S. D. Bader, Rev. Mod. Phys. 78, 1 (2006).

${ }^{4}$ C. Lamberti, Surf. Sci. Rep. 53, 1 (2004).
}

${ }^{5}$ M. Tolan, X-Ray Scattering from Soft-Matter Thin Films, Springer Tracts in Modern Physics Vol. 148 (Springer, Berlin, 1999).

${ }^{6}$ R. Röhlsberger, in Advances in Solid State Physics, edited by B. Kramer (Springer, Berlin, 2003), Vol. 43, pp. 529-544.

${ }^{7}$ P. Müller-Buschbaum, E. Bauer, E. Maurer, S. V. Roth, R. Gehrke, M. Burghammer, and C. Riekel, J. Appl. Crystallogr. 40, s341 (2006).

${ }^{8}$ R. Röhlsberger, H. Thomas, K. Schlage, E. Burkel, O. Leupold, and R. Rüffer, Phys. Rev. Lett. 89, 237201 (2002).

${ }^{9}$ R. L. Headrick, S. Kycia, Y. K. Park, A. R. Woll, and J. D. Brock, Phys. Rev. B 54, 14686 (1996).

${ }^{10}$ J.-P. Schlomka, M. Tolan, and W. Press, Appl. Phys. Lett. 76, 2005 (2000).

${ }^{11}$ T.-B. Hur, Y.-H. Hwang, H.-K. Kim, and H.-L. Park, J. Appl. Phys. 96, 1740 (2004).

${ }^{12}$ Y.-P. Wang, H. Zhou, L. Zhou, R. L. Headrick, A. T. Macrander, and A. S. Özcan, J. Appl. Phys. 101, 023503 (2007).

${ }^{13}$ L. Peverini, E. Ziegler, T. Bigault, and I. Kozhevnikov, Phys. Rev. B 72, 045445 (2005).

${ }^{14}$ W. Matz, N. Shell, W. Neumann, J. Bottiger, and J. Chevallier, Rev. Sci. Instrum. 72, 3344 (2001).

${ }^{15}$ D. Carbone, O. Plantevin, R. Gago, C. Mocuta, O. Bikondoa, A. Alija, L. Petit, H. Djazuli, and T.-H. Metzger, J. Synchrotron Radiat. 15, 414 (2008).

${ }^{16}$ E. Metwalli, S. Couet, K. Schlage, R. Röhlsberger, V. Körstgens, M. Ruderer, W. Wang, G. Kaune, S. V. Roth, and P. Müller-Buschbaum, Langmuir 24, 4265 (2008).

${ }^{17}$ T. Nakano and S. Baba, Vacuum 80, 647 (2006).

${ }^{18}$ M. T. Umlor, Appl. Phys. Lett. 87, 082505 (2005).

${ }^{19}$ R. M. A. Azzam and N. M. Bashara, Ellipsometry and Polarized Light (North-Holland, Amsterdam, 1986).

${ }^{20}$ G. S. D. Beach, F. T. Parker, D. J. Smith, P. A. Crozier, and A. E. Berkowitz, Phys. Rev. Lett. 91, 267201 (2003).

${ }^{21}$ Th. Diederich, S. Couet, and R. Röhlsberger, Phys. Rev. B 76, 054401 (2007).

${ }^{22}$ R. Röhlsberger, Nuclear Condensed Matter Physics with Synchrotron Radiation, Springer Tracts in Modern Physics Vol. 208 (Springer, Berlin, 2004).

${ }^{23}$ R. Rüffer and A. I. Chumakov, Hyperfine Interact. 97, 589 (1996).

${ }^{24}$ S.V. Roth, A. Rothkirch, G. Kaune, W. Wang, M.M. Abul Kashem, S. Couet, A. Diehert, R. Gehrke, H. Graafsma, M. Lohmann, E. Metwalli, M. Ruderer, A. Timmann, K. Schlage, R. Röhlsberger, and P. Müller-Buschbaum, HASYLAB Annual Report, 2007.

${ }^{25}$ C. Broennimann, E. F. Eikenberry, B. Henrich, R. Horisberger, G. Huelsen, E. Pohl, B. Schmitt, C. Schulze-Briese, M. Suzuki, T. Tomizaki, H. Toyokawa, and A. Wagner, J. Synchrotron Radiat. 13, 120 (2006).

${ }^{26}$ L. Tröger, D. Arvanitis, K. Baberschke, H. Michaelis, U. Grimm, and E. Zschech, Phys. Rev. B 46, 3283 (1992).

${ }^{27}$ A. Erbil, G. S. Cargill III, R. Frahm, and R. F. Boehme, Phys. Rev. B 37, 2450 (1988).

${ }^{28}$ C. H. Booth and F. Bridges, Phys. Scr., T 115, 202 (2005).

${ }^{29}$ B. Ravel and M. Newville, J. Synchrotron Radiat. 12, 537 (2005).

${ }^{30}$ S. Couet, K. Schlage, K. Saksl and R. Röhlsberger, Phys. Rev. Lett. 101, 056101(2008). 